\title{
Effect of temozolomide on the U-118 glioma cell line
}

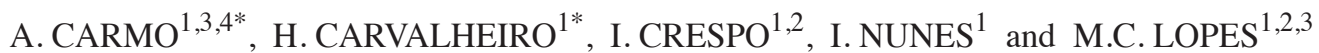 \\ ${ }^{1} \mathrm{CNC}$ - Center for Neuroscience and Cell Biology, University of Coimbra; ${ }^{2}$ Faculty of Pharmacy, University of \\ Coimbra; ${ }^{3}$ CIMAGO, University of Coimbra; ${ }^{4}$ University School of Vasco da Gama, Coimbra, Portugal
}

Received February 25, 2011; Accepted August 11, 2011

DOI: $10.3892 / \mathrm{ol} .2011 .406$

\begin{abstract}
Glioblastomas (GBM) are the most lethal subtype of astrocytomas, with a mean patient survival rate of 12 months after diagnosis. The gold standard treatment of GBM, which includes surgery followed by the combination of radiotherapy and chemotherapy with temozolomide (TMZ), increases the survival rate to 14.6 months. The success of TMZ appears to be limited by the occurrence of chemoresistance that allows glioma cells to escape from death signaling pathways. However, the mechanism of TMZ action is yet to be clarified although some controversial results have been reported. Therefore, our aim was to evaluate the occurrence of apoptosis and autophagy in glioma cells treated with TMZ and to correlate TMZ action with the survival pathways Pi3K/ Akt and ERK1/2 MAP kinase. Cell proliferation was evaluated by incorporation of bromodeoxyuridine. Apoptosis was studied by flow cytometry as well as by fluorescence confocal microscopy in order to evaluate the sub G0/G1 percentage of cells and chromatin condensation. The expression of the autophagy-associated protein, LC3, as well as Akt and ERK1/2 was performed by Western blotting. In TMZ-treated GBM cells the expression of LC3, the autophagy-associated protein was increased and only a reduced percentage of cells underwent apoptosis. In addition, we showed that the phosphorylation status of Pi3K/Akt and ERK1/2 MAP kinase was maintained during the treatment with TMZ, suggesting that glioma cells escape from TMZ-induced cell death due to these signaling pathways. The chemoresistance of U-118 cells to $\mathrm{TMZ}$ was partially eradicated when cells were simultaneously treated with specific inhibitors of Pi3K/Akt and ERK1/2 MAP kinase signaling pathways and TMZ. Therefore, we hypothesized that in order to induce glioma cell death it is essential to evaluate the activation of the survival pathways and establish a
\end{abstract}

Correspondence to: Dr Anália Carmo, Centre for Neuroscience and Cell Biology, IBILI, $1^{\circ}$ piso, Azinhaga de Santa Comba - Celas, 3000 Coimbra, Portugal

E-mail: analiacarmo@gmail.com

*Contributed equally

Key words: glioma, temozolomide, autophagy, apoptosis, ERK1/2, $\mathrm{Pi} 3 \mathrm{~K} / \mathrm{Akt}$ combined therapy using TMZ and inhibitors of those signaling pathways.

\section{Introduction}

Glioblastoma (GBM) is the most frequent type of glioma, comprising almost $50 \%$ of all diagnosed gliomas (1-4). The median survival of GBM patients remained between 9 and 12 months until 2004 when patients started chemotherapy with temozolamide (TMZ). In patients treated with $\mathrm{TMZ}$, the median survival increased to 14.6 months and the percentage of patients that had a 2-year survival rate increased to $17 \%$, as compared to that in patients not treated with TMZ (5). This improvement in median survival is extremely significant, but remains modest. The limited success of TMZ in GBM tumors appears to be related to the occurrence of chemoresistance and to the inability of TMZ to induce tumor cell death. TMZ is an alkylating agent that induces the formation of $\mathrm{O}^{6}$-methylguanine in DNA, which mispairs with thymine during the following cycle of DNA replication, leading to activation of the apoptotic pathways. In spite of this mechanism of action, Hirose et al reported that malignant glioma cells respond to TMZ by undergoing G2/M arrest and that few glioma cells treated with TMZ underwent apoptosis (6). In addition, Kanzawa et al found that TMZ induced autophagy, but not apoptosis in malignant glioma cells (7).

The mechanisms of TMZ action and the pathways by which glioma cells escape from death have yet to be adequately elucidated, and the reduced efficacy of TMZ in GBM treatment has yet to be determined. The reduced efficacy of TMZ in gliomas was initially attributed to the activity of MGMT which removes the DNA adducts. However, Hegi et al showed that even when the MGMT promoter was methylated, the median survival was 21.7 months (8). These results indicate that the mechanism of TMZ action may be overlapped by the survival signaling pathways. Previous studies reported that in patient tumor tissue samples the ERK1/2 and Pi3K/Akt were phosphorylated, indicating that these survival pathways were active in glioma cells $(7,9-12)$. Since these signaling pathways sustain key features that characterize gliomas, e.g., enhance proliferation and invasion, protect from proapoptotic stimuli and activate autophagy, it is likely that they may contribute to chemoresistance.

The activation status of cell survival pathways Pi3K/Akt, ERK1/2 and of autophagy in GBM cells treated with TMZ is poorly understood. Since TMZ is the first-line treatment 
in patients with GBM and 45\% of GBM patients are resistant to TMZ-treatment, the purpose of this study was to evaluate whether the activation status of Pi3K/Akt, ERK1/2 and autophagy interferes with the mechanism of action of TMZ.

\section{Materials and methods}

Reagents. DMEM, fetal bovine serum (FBS), propidium iodide (PI) and Hoechst were supplied by Invitrogen (Paisley, UK). The protease and phosphatase inhibitors were supplied by Roche (Indianapolis, IN, USA). Antibodies for Phospho-Akt, Phospho-ERK1/2 and total ERK1/2 were purchased from Cell Signalling Technology (MA, USA), the LC3 antibody was purchased from Affinity Bioreagents (Rockford, IL, USA) and mouse anti-actin antibody was purchased from Boehringer Mannheim (Germany). The phosphatase linked anti-mouse and anti-rabbit antibodies, and the substrate for the phosphatase were obtained from GE Healthcare (UK). PVDF membranes were purchased to Millipore (MA, USA). TMZ and the other chemicals were purchased from Sigma Chemicals (St. Louis, MO, USA). TMZ was dissolved in dimethyl sulfoxide (DMSO) at a concentration stock of $0.133 \mathrm{M}$. This stock was aliquoted and diluted with culture medium according to the used concentration. The bromodeoxyuridine (BrdUrd) kit to detect cell proliferation was purchased from Roche.

Cell line and cell culture conditions. The U-118 GBM cell line was purchased from the American Tissue Culture Collection, and maintained in Dulbecco's modified Eagle's medium (DMEM) supplemented with $3.5 \mathrm{mg} / \mathrm{ml}$ glucose, $0.1 \mathrm{mg} / \mathrm{ml}$ penicillin, $0.14 \mathrm{mg} / \mathrm{ml}$ streptomycin and $10 \%$ inactivated FBS. The cultured cells were maintained at $37^{\circ} \mathrm{C}$, in an atmosphere containing 95\% air and 5\% $\mathrm{CO}_{2}$. Cells were subcultured every $48 \mathrm{~h}$ by lifting them up with a cell scrapper. The cells were then centrifuged and resuspended in fresh DMEM. For the experiments, unsynchronized cells were treated with different concentrations of TMZ $(0,10,20,100$, 250 and $500 \mu \mathrm{M}$ ) for 24 and $48 \mathrm{~h}$. Assays were previously performed in the presence of DMSO, which corresponded to each TMZ concentration.

Cell proliferation assay. Cells were plated in 96 multi-well plates at different TMZ concentrations, for 24 and $48 \mathrm{~h}$. The effect of TMZ on the proliferation rate was assayed by a BrdUrd kit. Briefly, at the end of the incubation period $10 \mu \mathrm{l}$ of a BrdUrd solution in culture medium (1:100) was added to each well, and the cells were incubated for $6 \mathrm{~h}$. The culture medium was then removed by rapidly flicking it off the multiwell plate. The cells were fixed, incubated with an anti-BrdU antibody linked to a peroxidase and then incubated with a peroxidase substrate. The absorbance of the final solution was read in an ELISA plate reader at a 450-nm wavelength.

Cell cycle analysis by flow cytometry. Cells were plated in six multi-well plates and incubated with TMZ, wortmannin, U-0126 and rapamycin for 24 and $48 \mathrm{~h}$. At the end of the incubation period, cells were centrifuged at $1,500 \mathrm{rpm}$ for $10 \mathrm{~min}$, the culture medium was discarded and the pellet was resuspended in a solution of $70 \%$ ethanol and maintained overnight. The cells were then centrifuged at $1,500 \mathrm{rpm}$ for $10 \mathrm{~min}$, the pellet was resuspended and incubated for $1 \mathrm{~h}$ in the dark, at room temperature, in a solution of PBS containing $10 \mu \mathrm{l} / \mathrm{ml} \mathrm{PI}$ and $10 \mu \mathrm{l} / \mathrm{ml}$ RNAse (13). The PI fluorescence was measured on a FACScan flow cytometer (BD FACSCalibur ${ }^{\mathrm{TM}}$ ) and the data were gated to exclude cell debris and aggregates. Data were analyzed on WinMDI for Windows.

Staining of U-118 TMZ-treated cells with Hoescht 33258. Cells were plated in six multi-well plates and incubated with TMZ for 24 and $48 \mathrm{~h}$. After that, cells were washed in a PBS solution, centrifuged at $1,500 \mathrm{rpm}$ for $10 \mathrm{~min}$ and incubated for 15 min with a solution of methanol and acetone (1:1). Cells were then centrifuged at $1,500 \mathrm{rpm}$ for $10 \mathrm{~min}$, washed with PBS and incubated with $5 \mu \mathrm{g} / \mathrm{ml}$ Hoescht 33258 solution for $5 \mathrm{~min}$ at room temperature. After the incubation time, cells were washed and resuspended in PBS and mounted with Vectashield on glass slides. The images were captured under a Zeiss LSM 510 Meta confocal microscope at a magnification of $x 63$, and viewed on a Zeiss LSM image browser (Version 4.2.0.121; Carl Zeiss Inc., Germany).

Study of protein expression. Cells incubated with TMZ for 24 and $48 \mathrm{~h}$ were centrifuged at $1,500 \mathrm{rpm}$ for $10 \mathrm{~min}$ at $4^{\circ} \mathrm{C}$. The supernatant was discarded, cells were treated with RIPA buffer $(50 \mathrm{mM}$ Tris $\mathrm{HCl}$ at $\mathrm{pH} 8.0,150 \mathrm{mM} \mathrm{NaCl}, 1.0 \%$ NP-40, $0.5 \%$ sodium deoxycholate, $0.1 \%$ SDS and $2 \mathrm{mM}$ EDTA) supplemented with protease, phosphatase inhibitors and DTT, and cell suspensions were sonicated. The protein content of each sample was assessed and then proteins were denatured. Following that, the buffer (Tris $0.5 \mathrm{mM}, \mathrm{pH} 6.8$; $50 \%$ glycerol, $10 \%$ SDS, $10 \% 2 \beta$-mercaptoethanol and blue bromophenol) was added to the samples at a 1:1 ratio (14). Protein extracts were then boiled at $95^{\circ} \mathrm{C}$ for $5 \mathrm{~min}$ and used.

For Western blotting, $70 \mu \mathrm{g}$ of protein was separated on a $12 \%$ SDS-PAGE and then transferred to a PVDF membrane. The PVDF membrane was blocked with a solution of $5 \%$ milk in TBST for $1 \mathrm{~h}$ at room temperature and incubated overnight at $4^{\circ} \mathrm{C}$ with the primary antibody against LC3, p-Pi3K/Akt or p-ERK1/2 diluted in TBST with $1 \%$ milk supplemented with azide. Bound antibody was detected with an alkaline phosphatase conjugated anti-rabbit antibody, using enhanced chemifluorescence detection reagents. The protein expression was quantitated using the ImageQuant TL for Windows (version 2005; Amersham Biosciences, Piscataway, NJ, USA) with the expression of $\beta$-actin as a loading control for LC3, as well as total Pi3K/Akt and total ERK1/2 as a loading control for $\mathrm{p}-\mathrm{Pi} 3 \mathrm{~K} / \mathrm{Akt}$ and $\mathrm{p}$-ERK1/2, respectively.

Statistical analysis. Statistical analysis was performed on GraphPad Prism 5 for Windows (version 5.00; GraphPad Software, Inc., San Diego, CA, USA). Statistical significance within groups was assessed by a t-test and between groups by a two-way ANOVA, with a significance threshold of $p \leq 0.05$.

\section{Results}

$T M Z$ reduced $U-118$ proliferation. The effect of TMZ in U-118 cell proliferation was evaluated by quantification of the incorporated BrdUrd. TMZ treatment induced a significant 


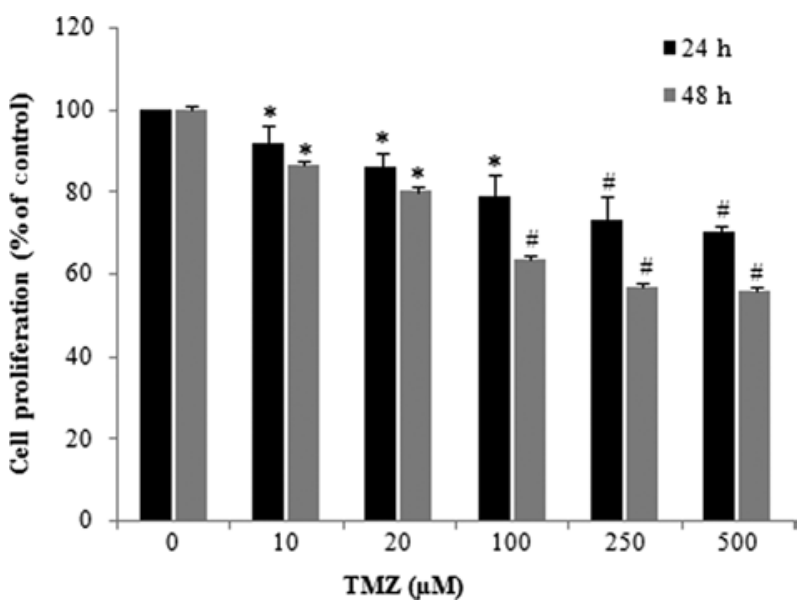

Figure 1. TMZ reduces glioma cell proliferation. U-118 cells were incubated for 24 and $48 \mathrm{~h}$ in the presence of different concentrations of TMZ. The proliferation rate was evaluated by measuring the incorporation of BrdUrd. Values are the means \pm SEM of triplicate analysis, each one performed in triplicate. ${ }^{*} \mathrm{p}<0.05$.

inhibition of the BrdUrd incorporation in glioma cells, which occurred in a dose- and time-dependent manner (Fig. 1). The effect of TMZ was particularly evident when U-118 cells were incubated with TMZ concentrations of $>100 \mu \mathrm{M}$. When U-118 cells were incubated with TMZ $(250 \mu \mathrm{M})$ the proliferation rate was inhibited by $43.2 \%$, as compared to that observed in the control cells.

Effect of TMZ on the cell cycle. To determine whether the reduced proliferation was related to an increase in apoptosis, the effect of TMZ in the cell cycle was also evaluated. The cell cycle of untreated cells was characterized by a long and well-defined G0/G1 peak, with a slightly prominent G2/M peak and a relatively low apoptotic fraction. When cells were incubated with TMZ, the percentage of cells in G0/G1 was reduced, the percentage of cells in sub G0/G1, which were considered as apoptotic cells, significantly increased and the percentage of cells in $\mathrm{S}$ and $\mathrm{G} 2 / \mathrm{M}$ was not significantly altered (Fig. 2). These alterations were more evident when cells were incubated for $48 \mathrm{~h}$ with TMZ. In the presence of $\mathrm{TMZ}(250 \mu \mathrm{M})$, the percentage of cells in the sub G0/G1 phase increased $15.4 \%$ and the percentage of cells in G0/G1 decreased $14.6 \%$, as compared to the control. These results indicated that TMZ had a reduced ability to induce apoptosis and also did not induce cell-cycle arrest in G1/S or in G2/M.

TMZ induced chromatin condensation. To evaluate whether TMZ induced morphological alterations compatible with apoptosis, U-118 cells were stained with Hoechst 33258 dye. When cells were incubated with TMZ, it was possible to detect chromatin condensation, irregular nuclei contour and pycnotic nuclei in certain cells confirming the results obtained from cell cycle analysis (Fig. 3). These observations were more evident when the TMZ concentration was $>100 \mu \mathrm{M}$, and also when cells were incubated with TMZ for $48 \mathrm{~h}$.

Expression of $L C 3$ in the presence of TMZ. Since there was a significant decrease in U-118 cell proliferation and the percentage of apoptotic cells was reduced, the expression

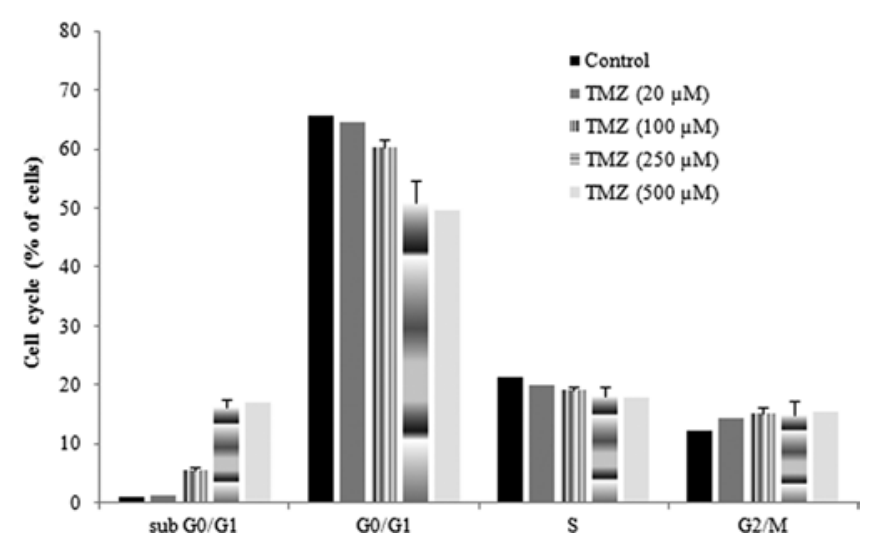

Figure 2. Cell cycle analysis of U-118 cells treated with TMZ (20, 100, 250 and $500 \mu \mathrm{M}$ ) for $48 \mathrm{~h}$. The results were obtained through the analysis of histograms and are representative of three experiments performed in triplicate. A total of 10,000 events were analyzed for each experiment. " $\mathrm{p}<0.01$.
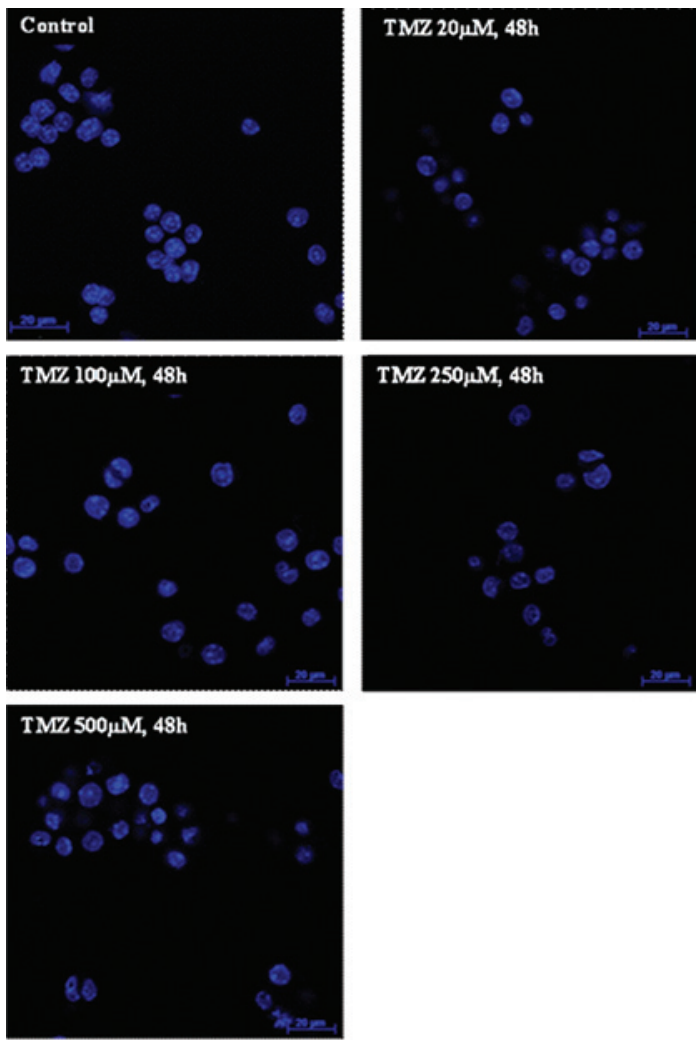

Figure 3. Confocal microscopy analysis of nuclear morphology by Hoechst 33258 staining of U-118 cells incubated with different concentrations of TMZ for a period of $48 \mathrm{~h}$. Magnification, x63.

of LC3, one of the autophagosome-membrane proteins, was analyzed. The results showed that U-118 cells constitutively expressed the LC3 protein. However, in U-118 cells incubated with TMZ there was an increase in the LC3 expression, which reached a maximum in the presence of $500 \mu \mathrm{M}$ of TMZ. This increase in LC3 expression was statistically significant when cells were incubated with TMZ for $48 \mathrm{~h}$ in the presence of 250 and $500 \mu \mathrm{M}$ (Fig. 4). These results may justify the reduction of proliferation in U-118 glioma cells incubated with TMZ, as well as the development of chemoresistance. 


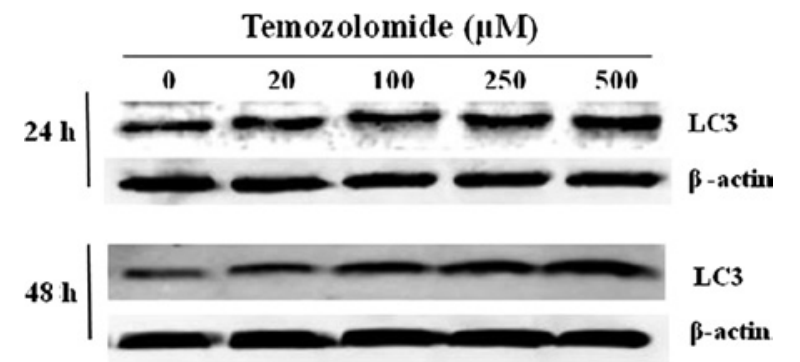

Figure 4. LC 3 expression. Following the incubation of U-118 cells with TMZ for $48 \mathrm{~h}$, the protein extracts were prepared and used for Western blot analysis with anti-LC3 antibody. Loading control was performed with an antibody for $\beta$-actin. The results are representative of three independent experiments.

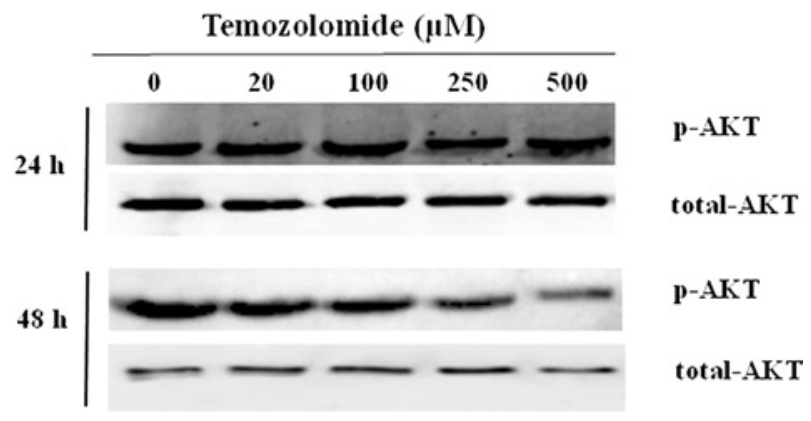

Figure 5. Akt phosphorylation status. Following the incubation of U-118 cells with TMZ for 24 and $48 \mathrm{~h}$, the protein extracts were prepared and used for Western blot analysis with anti-p-Akt antibody. Loading control was performed with an antibody for total-Akt. The results are representative of three independent experiments.

Role of Pi3K/Akt and ERK1/2 MAP kinase on U-118 cell survival. Since the induction of apoptosis is dependent on the DNA repair systems, as well as on the activity of various signaling pathways, the phosphorylation status of Pi3K/Akt and ERK1/2 MAP kinase was further evaluated by Western blotting. The results showed that the endogenous Akt was characterized by a basal phosphorylation of the Ser473 (Fig. 5). The basal level of p-Akt was maintained when cells were incubated for $24 \mathrm{~h}$, and it was slightly reduced when cells were incubated for $48 \mathrm{~h}$ with 250 and $500 \mu \mathrm{M}$ of TMZ. This reduction was not statistically significant and was probably associated with the increased apoptosis rate observed in the presence of TMZ (250 and $500 \mu \mathrm{M})$ for $48 \mathrm{~h}$.

The activation status of ERK1/2 MAP kinase was evaluated by determining the content of the p-ERK $1 / 2$ by Western blotting. The results showed that there was a basal activation of ERK1 and 2 in the U-118 glioma cells (Fig. 6). In the presence of TMZ, the amount of p-ERK1/2 was not significantly altered.

Induction of apoptosis in U-118 cells. Considering that the Pi3K/Akt and ERK1/2 MAP kinase were maintained in the phosphorylated forms in the presence of TMZ $(250 \mu \mathrm{M})$, and that these signaling pathways stimulate cellular proliferation, U-118 cells were incubated with wortmannin, the inhibitor of Pi3K/Akt; U-0126, the inhibitor of ERK1/2 MAP kinase and rapamycin, the inhibitor of mTOR. Cell cycle was then studied by flow cytometry, $48 \mathrm{~h}$ after the addition of the inhibitors, and the percentage of cells in the sub G0/G1 phase was quantified (Fig. 7).

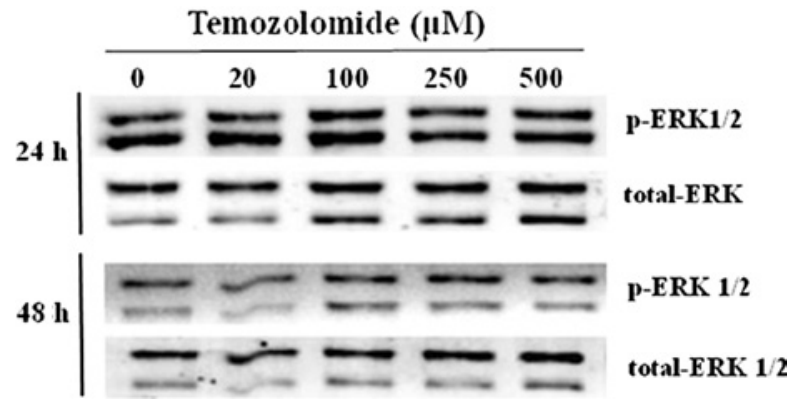

Figure 6. ERK1/2 phosphorylation status. Following the incubation of U-118 cells with TMZ for 24 and $48 \mathrm{~h}$, the protein extracts were prepared and used for Western blot analysis with anti-p-ERK1/2 antibody. Loading control was performed with an antibody for total ERK1/2. The results are representative of three independent experiments.

The results showed that the percentage of apoptotic cells in the presence of wortmannin was $18.24 \%$, which is similar to that observed in the presence of TMZ. However, when wortmannin was used in combination with TMZ, the percentage of apoptotic cells was $43.09 \%$, indicating that the inhibition of Pi3K/Akt contributes to the chemoresistance of glioma cells to TMZ.

When cells were incubated with rapamycin, the percentage of apoptotic cells increased to $26.5 \%$ as compared to that observed in the control cells, and was $11.13 \%$ higher than the percentage of apoptosis detected in the presence of TMZ. When cells were simultaneously incubated with rapamycin and TMZ, the percentage of apoptotic cells was $37.44 \%$, indicating that mTOR also contributes to U-118 cell survival.

Since mTOR is a downstream effector of Akt, U-118 cells were further incubated with wortmannin, rapamycin and TMZ. As shown in Fig. 7A, the percentage of apoptotic cells was found to be $63.01 \%$, indicating that the Pi3K/Akt/mTOR signaling pathways contribute to the chemoresistance of these glioma cells.

The contribution of ERK1/2 MAP kinase to glioma cell survival was evaluated in the presence of the U-0126. As shown in Fig. 7B, the percentage of apoptotic cells was 33.3\% in the presence of U-0126. When U-0126 was combined with TMZ the percentage of apoptotic cells was $51.6 \%$, an increase of $35.25 \%$ as compared to the percentage of apoptotic cells detected in the presence of TMZ (Fig. 7).

U-118 cells were then incubated with wortmannin, rapamycin, U-0126 and TMZ, and the cell cycle was evaluated. The percentage of apoptotic cells determined in this condition was $71.0 \%$, indicating that the survival of glioma cells depends on the activity of Akt/mTOR and ERK1/2 MAP kinase, and also that the blockage of the two signaling pathways contributes to an increase in the efficacy of TMZ.

\section{Discussion}

The EORTC/NCIC trial demonstrated an improvement in overall median survival of GBM patients from 12.1 months in patients submitted to radiotherapy, to 14.6 months in the TMZ-treated patients (15-18). This survival increment was extended by 6.4 months when the promoter of the MGMT was methylated $(8,11)$. As a consequence of these results, TMZ is 

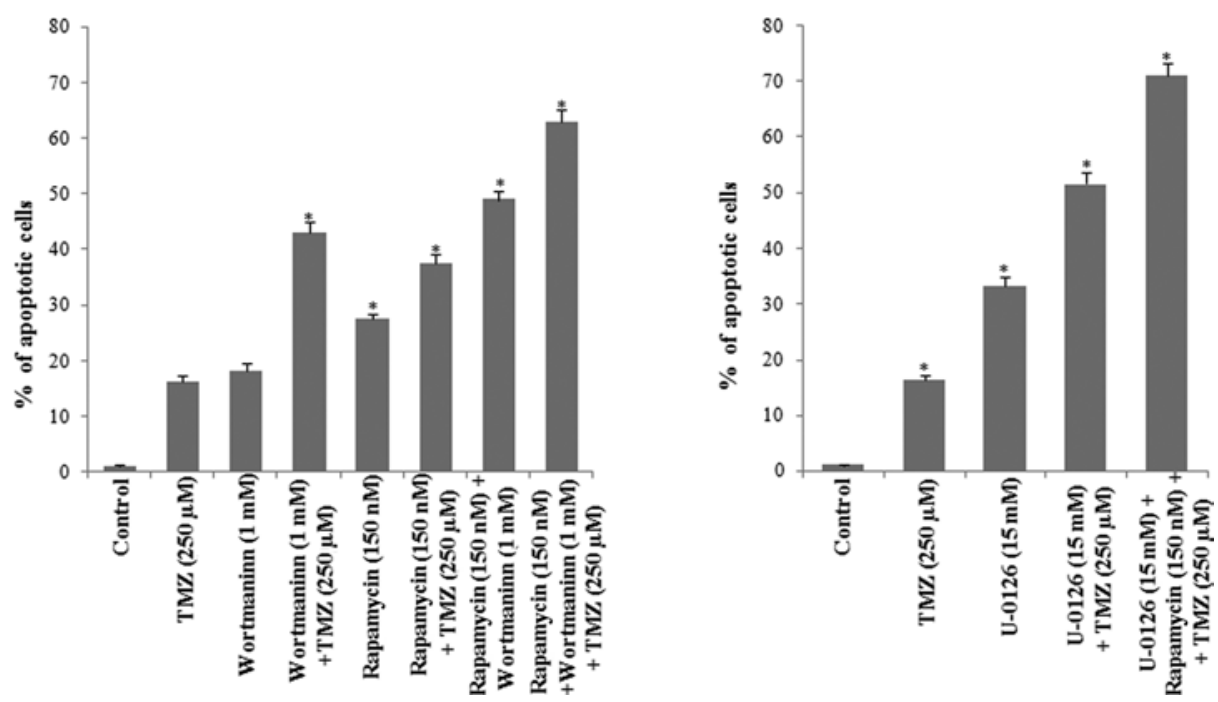

Figure 7. Cell cycle analysis of U-118 cells. (A) Cells treated with TMZ $(250 \mu \mathrm{M})$, wortmannin $(1 \mathrm{mM})$ and rapamycin $(150 \mathrm{nM})$ for $48 \mathrm{~h}$. (B) Cells treated with TMZ $(250 \mu \mathrm{M})$, rapamycin $(150 \mathrm{nM})$ and $\mathrm{U}-0126(15 \mathrm{mM})$ for $48 \mathrm{~h}$. The results were obtained through the analysis of histograms and are representative of three experiments performed in triplicate. A total of 10,000 events were analyzed for each experiment. "p $<0.01$ as compared to cells incubated with TMZ (250 $\mu \mathrm{M})$.

considered the gold standard in GBM treatment. However, the increment in survival remains reduced and therefore the study of the mechanism of action of TMZ is a fundamental issue.

The ability of alkylating agents to induce apoptosis is dependent not only on the MGMT activity, but also on the activity of various survival pathways. Thus, analysis of the TMZ effect on apoptosis, as well as the study of the interactions between TMZ and the survival pathways (Pi3K/Akt, ERK1/2 and autophagy), may contribute to improving the survival of GBM patients.

Regarding the mechanism of TMZ action, the results from previous studies were contradictory. Kanzawa et al showed that TMZ induced autophagy, but not apoptosis in malignant glioma cells (7). On the other hand, Hirose et al reported that in glioma cells TMZ induced a low level of apoptosis as compared to that observed in lymphoid cells, and that TMZ induced a cell cycle arrest in G2/M (19). In addition, Roos et al showed that cell death induced by TMZ in gliomas was due to apoptosis (20).

In our study, TMZ induced a significant increase in the expression of LC3, the protein associated with autophagosomes. The activation of autophagy may explain the reduction in U-118 proliferation observed in the presence of TMZ and may also constitute a survival mechanism of glioma cells. The signaling pathway of autophagy remains to be elucidated, since it may allow for the adaptation of tumor cells to adverse conditions induced by cancer therapy, contributing to tumor cell survival and consequently to chemoresistance (21-24). However, when irreversibly activated, autophagy triggers cell death, contributing to an increase in therapeutic efficacy (21-25). Therefore, understanding the mechanism involved in the activation of irreversibe autophagy may contribute to reducing the survival of glioma.

Our study also demonstrated that TMZ induced a low level of apoptosis that was not accompanied by cell cycle arrest. The reduced level of apoptosis may be associated with the activity of MGMT, Pi3K/Akt and/or ERK1/2 MAP kinase. Since the U-118 glioma cells are characterized by hypermethylation of the MGMT promoter (26), the reduced ability of
TMZ to induce apoptosis does not appear to be associated with the activity of this DNA repair enzyme. Therefore, we analyzed the phosphorylation of the signaling pathways Pi3K/ Akt and ERK1/2 MAP kinase.

The results indicated that Pi3K/Akt was constitutively active in the U-118 cells and that the active state was maintained in glioma cells treated with TMZ. Since a permanent phosphorylation of Pi3K/Akt may suppress the G2 checkpoint by altering activation of the DNA damage signal transducer Chk2 and the downstream effectors of the G2 checkpoint (12), we suggest that the active state of Pi3K/Akt contributed to the ability of U-118 cells to overcome the G2 checkpoint, thereby avoiding the effect of TMZ. Confirming the suppression of G2/M checkpoint is the fact that we did not observe any accumulation of cells in G2/M when cells were incubated with TMZ. To confirm the contribution of Pi3K/Akt to chemoresistance, we incubated U-118 cells with wortmannin, a specific inhibitor of Pi3K/Akt, and measured apoptosis. In the presence of wortmannin the percentage of apoptotic cells increased $17.24 \%$ as compared to the control cells, indicating that wortmanin induced a similar percentage of apoptosis as TMZ. However, in the presence of wortmannin and TMZ the percentage of apoptotic cells was $43.01 \%$, an increase of $26.7 \%$ as compared to cells treated with TMZ, confirming that Pi3K/Akt contributed to the survival and chemoresistance of U-118 cells to TMZ. Since wortmannin inhibits the activity of mTOR and previous results showed that proliferation associated with the activity of Pi3K/Akt signaling pathway is mediated by mTOR $(27,28)$, we evaluated the effect of rapamycin, the inhibitor of mTOR, on U-118 cell survival.

The results show that mTOR plays a crucial role in glioma cell survival, since the percentage of apoptosis was $26.5 \%$ in the presence of rapamycin. We also showed that the percentage of apoptotic cells increased to $37.44 \%$ when rapamycin was combined with TMZ, indicating the existence of a synergistic effect. In addition, we reported that the dual inhibition of Pi3K/mTOR in the presence of TMZ renders cells more susceptible to apoptosis and therefore may constitute a therapeutic target. 
Regarding the study of ERK1/2 MAP kinase activation, our results indicated that U-118 cells were characterized by a constitutive activation of this signaling pathway which was maintained in the presence of TMZ. To evaluate the contribution of ERK1/2 MAP kinase to U-118 survival, cells were incubated with the inhibitor U-0126 in the absence and presence of TMZ. The results showed that in the presence of the ERK1/2 MAP kinase inhibitor, the percentage of apoptotic cells was $33.3 \%$. When cells were treated with U-0126 combined with TMZ, the percentage of apoptotic cells was $51.6 \%$, indicating that this signaling pathway also contributes to the survival of U-118 cells.

Previous studies reported that the sustained activation of ERK1/ERK2 contributed to G1- to S-phase progression and protected cells from apoptotic signaling $(29,30)$. Taking into consideration that the activation of Pi3K/Akt inhibits the G2-M arrest $(6,12)$ and that ERK1/2 MAP kinase inhibits the arrest in G0/G1 $(18,19)$, we hypothesized that in order to induce GBM cell death the two signaling pathways should be blocked. In the presence of wortmannin, rapamycin, U-0126 and TMZ the percentage of apoptosis increased to $71.01 \%$, which evidences the existence of a synergistic effect between the survival pathways and TMZ. Our results emphasize the plasticity of glioma cells and their ability to override difficulties associated with the blocking of signaling pathways. This plasticity may justify the resistance of glioma cells to TMZ as well as the differences in GBM cell sensitivity to this alkylating agent. These results also suggest the need to combine traditional chemotherapy with molecular-based therapy, in particular therapy associated with signaling pathways.

In conclusion, our results indicate that TMZ by itself is not effective in inducing cell death, even in the U-118 cell line, which is hypermethylated. To overcome the resistance of U-118 cells to TMZ it appears essential to evaluate i) the activation status of the Pi3K/Akt, mTOR, ERK1/2 MAP kinase and autophagy, and ii) the synergistic effect between TMZ and the inhibitors of these signaling pathways. Therefore, we hypothesize that before glioma patients initiate TMZ therapy, it is necessary to evaluate the phosphorylation of the Pi3K/ Akt and ERK1/2 MAP kinase and prevent the activation of autophagy by TMZ.

\section{References}

1. Schwartzbaum JA, Fisher JL, Aldape KD and Wrensch M: Epidemiology and molecular pathology of glioma. Nat Clin Pract Neurol 2: 494-503, 2006.

2. Ohgaki H and Kleihues P: Epidemiology and etiology of gliomas. Acta Neuropathol 109: 93-108, 2005.

3. CBTRUS, Statistical Report: Primary Brain Tumors in the United States, 2004-2006. Central Brain Tumor Registry of the United States, pp1-65, 2010.

4. Hadjipanayis CG and van Meir EG: Brain cancer propagating cells: biology, genetics and targeted therapies. Trends Mol Med 15: 519-530, 2009.

5. Stupp R, Mason WP, van den Bent MJ, et al: Radiotherapy plus concomitant and adjuvant temozolomide for glioblastoma. N Engl J Med 352: 987-996, 2005.

6. Hirose Y, Berger MS and Pieper RO: Abrogation of the Chk1-mediated $\mathrm{G}(2)$ checkpoint pathway potentiates temozolomide-induced toxicity in a p53-independent manner in human glioblastoma cells. Cancer Res 61: 5843-5849, 2001.

7. Kanzawa T, Germano IM, Komata T, Ito H, Kondo Y and Kondo S: Role of autophagy in temozolomide-induced cytotoxicity for malignant glioma cells. Cell Death Differ 11: 448-457, 2004.
8. Hegi M, Diserens A, Gorlia T, et al: MGMT gene silencing and benefit from temozolomide in glioblastoma. N Engl J Med 352: 997-1003, 2005.

9. Keating AK, Kim GK, Jones AE, Donson AM, Ware K, Mulcahy JM, Salzberg DB, Foreman NK, Liang X, Thorburn A and Graham DK: Inhibition of Mer and Axl receptor tyrosine kinases in astrocytoma cells leads to increased apoptosis and improved chemosensitivity. Mol Cancer Ther 9: 1298-1307, 2010.

10. Akhavan D, Cloughesy TF and Mischel PS: mTOR signaling in glioblastoma: lessons learned from bench to bedside. Neuro Oncol 12: 882-889, 2010.

11. Mrugala MM and Chamberlain MC: Mechanisms of disease: temozolomide and glioblastoma - look to the future. Nat Clin Pract Oncol 5: 476-486, 2008.

12. Hirose Y, Katayama M, Mirzoeva OK, Berger MS and Pieper RO: Akt activation suppresses Chk2-mediated, methylating agent-induced G2 arrest and protects from temozolomide-induced mitotic catastrophe and cellular senescence. Cancer Res 65: 4861-4869, 2005.

13. Carmo A, Patricio I, Cruz MT, Carvalheiro H and Lopes MC: CXCL12/CXCR4 promotes motility and proliferation of glioma cells. Cancer Biol Ther 9: 56-65, 2010.

14. Neves BM, Cruz MT, Francisco V, Goncalo M, Figueiredo A, Duarte CB and Lopes MC: Differential modulation of CXCR4 and CD40 protein levels by skin sensitizers and irritants in the FSDC cell line. Toxicol Lett 177: 74-82, 2008.

15. Van Meir EG, Hadjipanayis CG, Norden AD, Shu H, Wen PY and Olson JJ: Exciting new advances in neuro-oncology: the avenue to a cure for malignant glioma. CA Cancer J Clin 60: 166-193, 2010.

16. Sathornsumetee S, Reardon DA, Desjardins A, Quinn JA, Vredenburgh JJ and Rich JN: Molecularly targeted therapy for malignant glioma. Cancer 110: 13-24, 2007.

17. Furnari F, Fenton T, Bachoo R, et al: Malignant astrocytic glioma:genetics, biology, and paths to treatment. Genes and Dev 21: 2683-2710, 2007.

18. Gladson CL, Prayson RA and Liu WM: The pathobiology of glioma tumors. Annu Rev Pathol Mech Dis 5: 33-50, 2010.

19. Hirose Y, Berger MS and Pieper RO: p53 effects both the duration of $\mathrm{G} 2 / \mathrm{M}$ arrest and the fate of temozolomide-treated human glioblastoma cells. Cancer Res 61: 1957-1963, 2001.

20. Roos WP, Batista LF, Naumann SC, Wick W, Weller M, Menck CF and Kaina B: Apoptosis in malignant glioma cells triggered by the temozolomide-induced DNA lesion O6-methylguanine. Oncogene 26: 186-197, 2007.

21. Ogier-Denisy E and Codogno P: Autophagy: a barrier or an adaptive response to cancer. Biochim Biophys Acta 1603: 113-128, 2003.

22. Mariño G and López-Otín C: Autophagy: molecular mechanisms, physiological functions and relevance in human pathology. Cell Mol Life Sci 61: 1439-1454, 2004.

23. Fu J, Shao C, Chen F, Ng H and Chen Z: Autophagy induced by valproic acid is associated with oxidative stress in glioma cell lines. Neuro Oncol 12: 328-340, 2009.

24. Thorburn A: Apoptosis and autophagy: regulatory connections between two supposedly different processes. Apoptosis 13: 1-9, 2008.

25. Katayama M, Kawaguchi T, Berger MS and Pieper RO: DNA damaging agent-induced autophagy produces a cytoprotective adenosine triphosphate surge in malignant glioma cells. Cell Death Differ 14: 548-558, 2007.

26. Lorente A, Mueller W, Urdangarín E, Lázcoz P, Deimling A and Castresana JS: Detection of methylation in promoter sequences by melting curve analysis-based semiquantitative real time PCR. BMC Cancer 8: 61-75, 2008.

27. Ronellenfitsch MW, Brucker DP, Burger MC, Wolking S, Tritschler F, Rieger J, Wick W, Weller M and Steinbach JP: Antagonism of the mammalian target of rapamycin selectively mediates metabolic effects of epidermal growth factor receptor inhibition and protects human malignant glioma cells fromhypoxia-induced cell death. Brain 13: 1509-1522, 2009.

28. Law BK: Rapamycin: an anti-cancer immunosuppressant? Crit Rev Oncol Hematol 56: 47-60, 2005.

29. Meloche S and Pouyssegur J: The ERK1/2 mitogen-activated protein kinase pathway as a master regulator of the G1- to S-phase transition. Oncogene 26: 3227-3239, 2007.

30. Tran SE, Holmstrom TH, Ahonen M, Kahari VM and Eriksson JE: MAPK/ERK overrides the apoptotic signaling from Fas, TNF, and TRAIL receptors. J Biol Chem 276: 16484-16490, 2001. 